\title{
Interstellar absorption lines toward Cep OB4^
}

\author{
R. Gredel
}

\begin{abstract}
Max-Planck Institut für Astronomie (MPIA), Königstuhl 17, 69117 Heidelberg, Germany
e-mail: gredel@mpia.de; gredel@caha.es
\end{abstract}

Received 29 March 2004 / Accepted 10 June 2004

\begin{abstract}
An analysis of optical echelle spectra towards nine stars in the Cep OB4 association is presented. Interstellar absorption lines which arise in the $(1,0)$ and $(2,0)$ bands of the $\mathrm{CN} \mathrm{A}{ }^{2} \Pi-\mathrm{X}^{2} \Sigma^{+}$red system towards $\mathrm{BD}+66^{\circ} 1661, \mathrm{BD}+66^{\circ} 1674$, and $\mathrm{BD}+66^{\circ} 1675$ are used to infer accurate $\mathrm{CN}$ column densities $N(\mathrm{CN})$. A comparison with earlier measurements in the $\mathrm{CN}$ violet system allows to infer a CN Doppler $b$ parameter of $b(\mathrm{CN})=1.2-2.4 \mathrm{~km} \mathrm{~s}^{-1}$. Molecular carbon absorption lines which arise in the $(1,0),(2,0)$ and $(3,0)$ bands of the $\mathrm{C}_{2} \mathrm{~A}^{1} \Pi_{\mathrm{u}}-\mathrm{X}^{1} \Sigma_{\mathrm{g}}^{+}$Phillips system is used to infer gaskinetic temperatures of $35 \pm 10 \mathrm{~K}$ and densities of $n=700 \pm 200 \mathrm{~cm}^{-3}$ towards $\mathrm{BD}+66^{\circ} 1661$ and $\mathrm{BD}+66^{\circ} 1675$, and a temperature of $60 \pm 10 \mathrm{~K}$ and a density of $800 \pm 400 \mathrm{~cm}^{-3}$ towards $\mathrm{BD}+66^{\circ} 1674$. The $\mathrm{R}(1)$ line of the $(0,0)$ band of the $\mathrm{CH} \mathrm{A} \mathrm{A}^{2} \Delta-\mathrm{X}^{2} \Pi$ system is detected towards 6 stars. A tight correlation exists between $N\left(\mathrm{C}_{2}\right)$ and $N(\mathrm{CH}) . N(\mathrm{CN})$ increases with $N(\mathrm{CH})$ and with $N\left(\mathrm{C}_{2}\right)$. Interstellar $\mathrm{CH}^{+}$ is marginally detected towards four stars. Inferred $\mathrm{CH}^{+}$column densities are significantly lower than towards other lines of sight with similar reddening. The velocity structure towards Cep OB4 and the chemical abundances suggest that $\mathrm{CN}, \mathrm{C}_{2}, \mathrm{CH}$, and $\mathrm{CH}^{+}$are formed in quiescent material. A previous suggestion that the molecules form in a photon-dominated region close to the stars is not supported by the observations.
\end{abstract}

Key words. ISM: abundances - ISM: clouds - ISM: molecules - ISM: lines and bands

\section{Introduction}

From his photographic observations of high-resolution spectra towards $\mathrm{BD}+66^{\circ} 1661, \mathrm{BD}+66^{\circ} 1674$, and $\mathrm{BD}+66^{\circ} 1675$ in the Cep OB4 association, Guido Münch (1964) detected the presence of very strong interstellar CN absorption lines towards these three heavily reddened early type supergiants. He speculated that the high $\mathrm{CN}$ abundance results from $\mathrm{CN}$ formation on grain surfaces, followed by a release of the molecules into the gas phase. The release is caused by an ionisation front which arises from the stars of Cep OB4. This scenario, which requires the presence of interstellar material near the stars, was supported by an earlier finding by Blanco \& Williams (1959). The authors noted that the intrinsically bluer stars are more reddened, and suggested that the younger stars are still surrounded by the gas and dust from the parent molecular cloud from which they have formed. While this unusual reddening effect was later assigned by Walker (1965) to arise from a selection effect, evidence for the presence of an HII- $\mathrm{H}_{2}$ interaction region towards Cep OB4 does in fact arise from millimeter CO observations (Yang \& Fukui 1992). The analysis of IRAS data by Luo (1995) also suggests that the strong ionising radiation and wind from Berkeley 59, the small central cluster of Cep OB4, have caused

* Based on observations collected at the Centro Astronómico Hispano Alemán (CAHA) at Calar Alto, operated jointly by the MaxPlanck Institut für Astronomie and the Instituto de Astrofisica de Andalucia (CSIC). the formation of a blister HII region into the natal molecular cloud.

The previous observations suggest that a $\mathrm{HII}-\mathrm{H}_{2}$ interaction region is located towards Cep OB4 and that it may be studied by traditional optical absorption line techniques. We have thus decided to perform a more comprehensive search of interstellar molecular absorption lines towards the Cep OB4 association. High signal-to-noise spectra towards nine stars in Cep OB4 were obtained using the echelle spectrograph at the Calar Alto $2.2 \mathrm{~m}$ telescope. The main aim here was to search for interstellar absorption lines of $\mathrm{CN}, \mathrm{C}_{2}, \mathrm{CH}$, and $\mathrm{CH}^{+}$in the spectra of the background stars. In general, observations of these molecules allow to infer physical parameters such as gaskinetic temperatures, densities, Doppler- $b$ values, and accurate molecular column densities. The observations are described in Sect. 2. An analysis of the results is given in Sect. 3, with an emphasis of the rotational excitation of $\mathrm{CN}$ and of $\mathrm{C}_{2}$ given in Sects. 3.2 and 3.3, respectively. The velocity structure of the region is discussed in Sect. 4.1. The inferred molecular abundances are compared with the expectations from models of photon-dominated regions (PDRs) and of quiescent translucent clouds in Sect. 4.3. A summary of the findings is given in Sect. 5.

\section{Observations and results}

Optical spectra of stars in the Cep OB4 association were obtained with the Calar Alto $2.2 \mathrm{~m}$ telescope using the fibre-fed 
Table 1. Observed stars and properties.

\begin{tabular}{lcrrrr}
\hline \hline Star & Spectral type & $\begin{array}{r}V^{a} \\
\text { mag }\end{array}$ & $\begin{array}{r}E_{B-V}^{a} \\
\text { mag }\end{array}$ & Obs. dates & $\begin{array}{r}t_{\text {int }} \\
\text { min }\end{array}$ \\
\hline BD+66 1661 & $O 9 V$ & 8.76 & 1.11 & Sep. 99 & $3 \times 20$ \\
& & & & Sep. 01 & $4 \times 20$ \\
BD+66 1673 & $B 0$ & 10.0 & 1.62 & Jan. 02 & $8 \times 20$ \\
$\mathrm{BD}+66^{\circ} 1674$ & $B 0$ & 9.63 & 0.73 & Sep. 99 & $6 \times 20$ \\
& & & & Sep. 01 & $6 \times 20$ \\
$\mathrm{BD}+66^{\circ} 1675$ & $O 7$ & 9.08 & 1.52 & Sep. 99 & $4 \times 20$ \\
& & & & Sep. 01 & $4 \times 20$ \\
$\mathrm{BD}+66^{\circ} 1669 \mathrm{a}$ & $B 1$ & 11.08 & 0.82 & Jan. 02 & $4 \times 20$ \\
$\mathrm{BD}+66^{\circ} 1669 \mathrm{~b}$ & $B 1$ & 10.76 & 1.0 & Jan. 02 & $4 \times 20$ \\
$\mathrm{BD}+67^{\circ} 1580$ & $B 3$ & 10.17 & 0.75 & Jan. 02 & $3 \times 20$ \\
$\mathrm{BD}+67^{\circ} 1585$ & $B 5$ & 10.33 & 0.92 & Jan. 02 & $8 \times 20$ \\
$\mathrm{BD}+67^{\circ} 1598$ & $B 2$ & 9.12 & 1.65 & Jan. 02 & $8 \times 20$ \\
$\mathrm{BD}+65^{\circ} 1973^{\dagger}$ & $B 2$ & 10.55 & $0.91^{b}$ & Jan. 02 & $8 \times 20$ \\
\hline
\end{tabular}

${ }^{a}$ Spectral type and magnitude from SIMBAD database; $E_{B-V}$ from McDonnell et al. (1968).

${ }^{b}$ Reddening from Blanco \& Williams (1959).

${ }^{\dagger}$ HIP 118192; not associated with Cep OB4 as inferred from Hipparcos proper motion and parallax as given by de Zeeuw et al. (1999).

echelle spectrograph FOCES. The instrument is described by Pfeiffer et al. (1998). FOCES was used with a $180 \mu \mathrm{m}$ entrance slit and a $15 \mu \mathrm{m} 2048 \times 2048$ pixel LORAL detector. This setup provided an actual 2 pixel resolution of $R=\lambda / \Delta \lambda=$ 60000 as judged from the width of the thorium lines. The large format detector allows to record the full optical spectrum from 3500-11000 $\AA$ in a single exposure. The observations were carried out during September 1999, September 2001, and January 2002. Observations in September 2001 and January 2002 were affected by poor weather. In Table 1, the observed stars, their spectral type, the visual magnitude $V$, the reddening $E_{B-V}$, the dates of the observations, and the total integration times $t_{\text {int }}$ are listed. With the exception of $\mathrm{BD}+66^{\circ} 1973$, the stars listed in Table 1 all have similar Hipparcos parallaxes and proper motions (de Zeeuw et al. 1999). The corresponding distances of $800-1000 \mathrm{pc}$ are consistent with the earlier determined distance of $850 \mathrm{pc}$ derived by MacConnell (1968). The star BD+66 1973 has been discarded as a member of the Cep OB4 association on the basis of its Hipparcos proper motion and parallax (de Zeeuw et al. 1999).

The data were reduced within the MIDAS echelle context. From the raw data frames, the background, which includes the bias and the scattered light level, was determined from a twodimensional fit to the inter-order regions. Flat-field corrections were achieved using integrations on an internal halogen lamp. The design of FOCES is such that the optical path of the light from the halogen lamp coincides very well with that of the stars, so very accurate flat-field corrections can be obtained using internal lamps (Grupp 2003). The location of the orders in the raw data files were determined using a Hough transformation. Individual orders were extracted using a 4-pixel slit. The spectra were rebinned to a linear, heliocentric wavelength scale using Th-Ar reference frames. Barycentric corrections were calculated within MIDAS. In the limit of unsaturated lines (Doppler parameters $b \rightarrow \infty$ ), the measured equivalent widths $W_{\lambda}$ are converted into column densities $N_{\infty}$ using $N_{\infty}=1.13 \times 10^{20} W_{\lambda} /\left(f_{J^{\prime} J^{\prime \prime}} \lambda^{2}\right)$, where $W_{\lambda}$ and wavelengths $\lambda$ are in units of $\AA$ and $N_{\infty}$ is in units of $\mathrm{cm}^{-2}$, and where $f_{J^{\prime} J^{\prime \prime}}$ are the line oscillator strengths.

\subsection{The $(1,0)$ and $(2,0)$ bands of the $A^{2} \Pi-\mathrm{X}^{2} \Sigma^{+} C N$ red system}

Generally, optical absorption line studies of interstellar $\mathrm{CN}$ focus the $(0,0)$ band of the $\mathrm{CN} \mathrm{B}{ }^{2} \Sigma^{+}-\mathrm{X}^{2} \Sigma^{+}$violet system, around $3875 \AA$. This is because the absorption lines in the violet system are strong and the corresponding spectral region is not contaminated by telluric absorption lines. For the heavily reddened stars observed here, the signal-to-noise ratio below $4000 \AA$ is too low to infer reliable equivalent widths. We thus proceed to analyse absorption lines which arise in the $(1,0)$ and $(2,0)$ bands of the $\mathrm{CN} \mathrm{A}^{2} \Pi-\mathrm{X}^{2} \Sigma^{+}$red system, at wavelengths around $9150 \AA$ and $7900 \AA$, respectively. $\mathrm{CN}$ lines in the $(3,0)$ band of the red system, around $6950 \AA$, are not detected in our spectra. The band oscillator strengths of the $\mathrm{CN}$ red system are almost an order of magnitude lower than those of the $\mathrm{CN}$ violet system, which allows in general a more accurate determination of column densities as saturation effects are less pronounced (e.g. Gredel et al. 1991). This advantage is somewhat impaired, however, by the fact that the absorption lines appear in spectral regions which are heavily contaminated by telluric absorption lines.

Figure 1 contains the spectra of three stars where absorption lines in the $\mathrm{CN}$ red system are detected. For each of the three stars $\mathrm{BD}+66^{\circ} 1675, \mathrm{BD}+66^{\circ} 1674$, and $\mathrm{BD}+66^{\circ} 1661$, the spectral regions around $7875 \AA$, $7906 \AA$, and $9185 \AA$, are shown. Detected $\mathrm{CN}$ lines are marked by filled triangles. The detected lines all arise from the $J^{\prime \prime}=0$ level. Open triangles indicate the expected position of absorption lines which arise from the $J^{\prime \prime}=1$ level. $\mathrm{CN}$ absorption lines which arise from $J^{\prime \prime}+1$ are not detected in any of the spectra obtained here. All spectra are rebinned to heliocentric wavelength scales which causes shifts in the position of the telluric features from one star to the other. Figure 1 also contains, at the top, spectra towards $16 \mathrm{Tau}$, which do not contain $\mathrm{CN}$ absorption lines. The comparison spectra of 16 Tau may serve to better illustrate the presence of $\mathrm{CN}$ absorption lines in the spectra of the other three stars.

A comprehensive discussion of the analysis of $\mathrm{CN}$ absorption lines which arise in the red system is given in Gredel et al. (1991). We use their listed wavelengths and band oscillator strengths to infer heliocentric velocities and column densities $N(0)$ in rotational level $J^{\prime \prime}=0$. We assume that saturation effects are negligible for the absorption lines detected in the $\mathrm{CN}$ red system. A summary of the measurements is given in Table 2, which contains, in Cols. 1-5, the line 

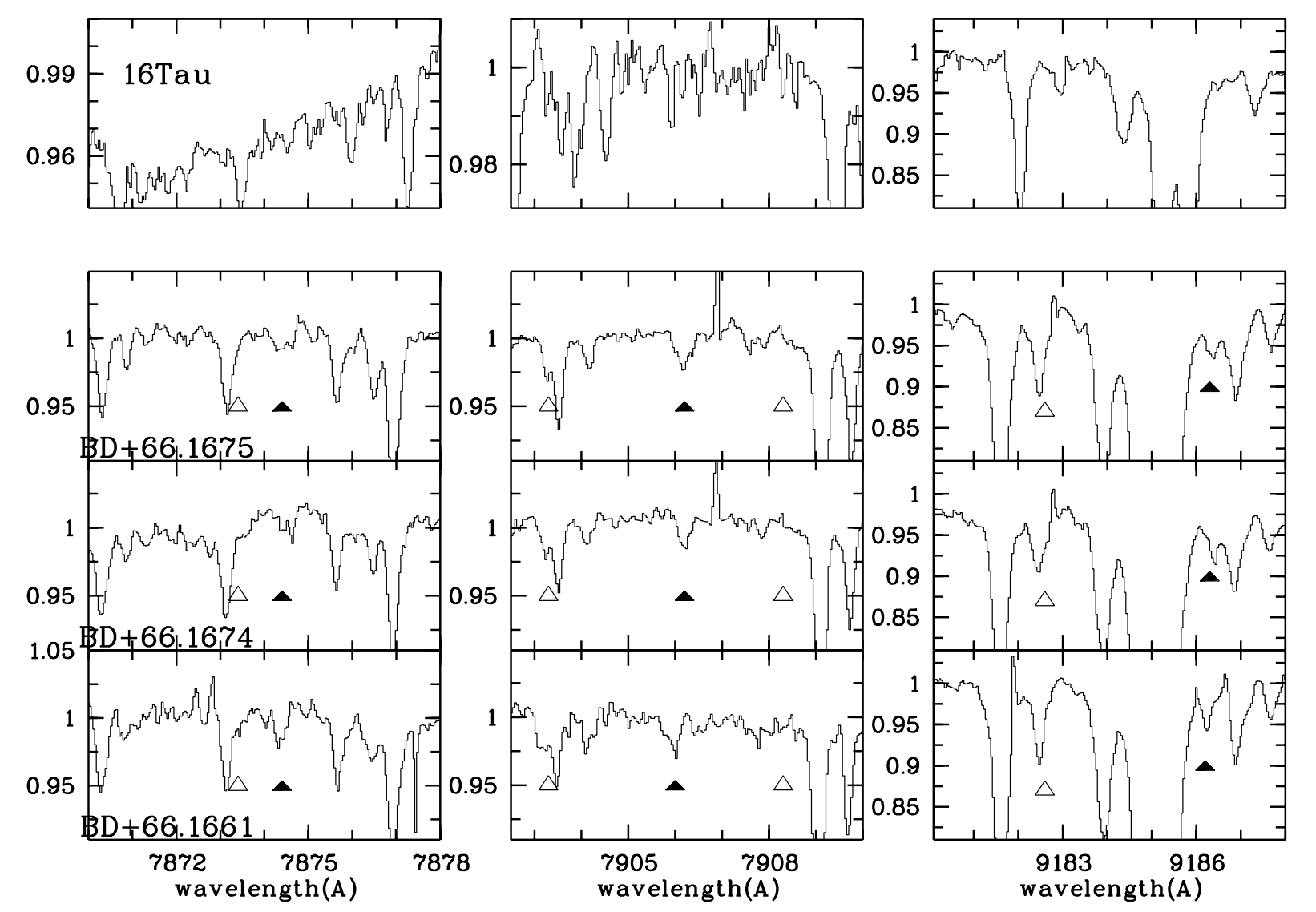

Fig. 1. Normalised spectra of interstellar $\mathrm{CN}$ lines of the $(1,0)$ and $(2,0)$ bands of the $\mathrm{A}{ }^{2} \Pi-\mathrm{X}^{2} \Sigma^{+}$red system towards $\mathrm{BD}+66^{\circ} 1661$, $\mathrm{BD}+66^{\circ} 1674$, and $\mathrm{BD}+66^{\circ} 1675$. The position of detected absorption lines due to $\mathrm{CN}$ are marked by filled triangles. Open triangles indicate the position of absorption lines arising from rotational level $J^{\prime \prime}=1$. Features not marked are due to telluric absorption lines. Spectra of 16 Tau which do not contain $\mathrm{CN}$ are shown for comparison.

identifications, the heliocentric wavelengths $\lambda_{\text {hel }}$, the measured equivalent widths $W_{\lambda}$, the inferred heliocentric velocities $V_{\text {hel }}$, and the column densities $N(0)$ in units of $10^{12} \mathrm{~cm}^{-2}$. Measurement uncertainties in $W_{\lambda}$ and corresponding uncertainties in $N(0)$ are given in parentheses.

Towards $\mathrm{BD}+66^{\circ} 1661$, the $(2,0) R_{1}(0)$ line near $7906 \AA$ is rather broad and may be decomposed into two velocity components near $V_{\text {hel }}=-22 \mathrm{~km} \mathrm{~s}^{-1}$ and $-29 \mathrm{~km} \mathrm{~s}^{-1}$, but the Gaussian fit is not unique. Toward $\mathrm{BD}+66^{\circ} 1675$, both the $(2,0) R_{1}(0)$ and $(2,0){ }^{R} \mathrm{Q}_{21}(0)$ lines, near $7906 \AA$ and $7874 \AA$, respectively, are decomposed into three absorption components near $-22.5 \mathrm{~km} \mathrm{~s}^{-1},-16.5 \mathrm{~km} \mathrm{~s}^{-1}$, and $-9.3 \mathrm{~km} \mathrm{~s}^{-1}$, but again, the fit is not unique. The $(1,0) R_{1}(0)$ towards $\mathrm{BD}+66^{\circ} 1675$ is blended with a telluric absorption line, and the listed velocity of $-0.4 \mathrm{~km} \mathrm{~s}^{-1}$ may not be accurate. Towards BD $+66^{\circ} 1673$, the $(1,0) R_{1}(0)$ line is blended with a telluric absorption line and the listed equivalent width is highly uncertain. The latter two measurements are ignored in the discussion of Sect. 4.

\section{2. $\mathrm{CH}$ and $\mathrm{CH}^{+}$absorption lines}

Towards most of the stars of the present sample, strong interstellar absorption lines of $\mathrm{CH}$ which arise from the $R_{2 \mathrm{e}}(1)+$ $R_{2 \mathrm{f}}(1) \Lambda$-doublet of the $(0,0)$ band of the $\mathrm{A}^{2} \Delta-\mathrm{X}^{2} \Pi$ system are detected. The corresponding spectra are reproduced in Fig. 2.
The star $\mathrm{BD}+66^{\circ} 1669$ is a visual double star and the spectra towards both components are shown. The $\mathrm{CH}$ absorption lines towards the stars are all unresolved, but the spectra indicate the presence of multiple velocity components. This is for instance seen towards $\mathrm{BD}+66^{\circ} 1598$ and $\mathrm{BD}+66^{\circ} 1661$, where red wings appear in the $\mathrm{CH}$ line profile. A robust decomposition into individual Gaussians is not possible. A decomposition of the $\mathrm{CH}$ line profile toward $\mathrm{BD}+66^{\circ} 1675$ into the three velocity components seen in CN (cf. Sect. 2.1) is possible, but it is not unique. In the major part of the discussion below, we use integrated equivalent widths and centroid wavelengths only.

The measured equivalent widths are rather large, which makes the derived column densities uncertain because of possible saturation effects. The level of saturation can be determined from a comparison with absorption lines which arise in the $\mathrm{CH} \mathrm{B}{ }^{2} \Sigma^{-}-\mathrm{X}^{2} \Pi$ system, which have oscillator strengths about a factor of two lower. Absorption lines of the B-X system are, however, not detected in our spectra. We proceed and adopt a value of $b(\mathrm{CH})=1 \mathrm{~km} \mathrm{~s}^{-1}$ to estimate the magnitude of saturation effects. Very high spectral resolution observations of Andersson et al. (2002) and Crawford (1995), among others, show that Doppler values around $b \approx 1 \mathrm{~km} \mathrm{~s}^{-1}$ are very typical indeed for individual velocity components of $\mathrm{CH}$. The curve of growth analysis used to infer column densities is described in Gredel et al. (1993). In the analysis, the $\Lambda$-doubling of 
Table 2. Measured CN lines.

\begin{tabular}{|c|c|c|c|c|}
\hline $\begin{array}{l}\text { Star and } \\
\text { line ID }\end{array}$ & $\begin{array}{c}\lambda_{\text {hel }} \\
\AA\end{array}$ & $\begin{array}{r}W_{\lambda} \\
\mathrm{m} \AA\end{array}$ & $\begin{array}{r}V_{\text {hel }} \\
\mathrm{km} \mathrm{s}^{-1}\end{array}$ & $\begin{array}{r}N(0) \\
10^{12} \mathrm{~cm}^{-2}\end{array}$ \\
\hline \multicolumn{5}{|l|}{$\mathrm{BD}+67^{\circ} 1598$} \\
\hline$(2,0) R_{1}(0)$ & 7906.228 & $1.8(0.5)$ & -14.1 & $7.9(2.3)$ \\
\hline \multicolumn{5}{|l|}{$\mathrm{BD}+66^{\circ} 1661$} \\
\hline$(2,0) R_{1}(0)$ & $7905.831^{a}$ & $3.8(0.5)$ & -29.2 & $17.1(2.3)$ \\
\hline$(2,0) R_{1}(0)$ & $7906.015^{a}$ & $3.8(0.5)$ & -22.2 & $17.1(2.3)$ \\
\hline$(2,0)^{R} Q_{21}(0)$ & 7874.351 & $5.3(0.5)$ & -18.9 & 38.1(3.6) \\
\hline$(1,0) R_{1}(0)$ & 9186.235 & $17(10)$ & -22.8 & $28.7(16.9)$ \\
\hline \multicolumn{5}{|l|}{$\mathrm{BD}+66^{\circ} 1673$} \\
\hline$(2,0) R_{1}(0)$ & 7906.269 & $4.4(0.5)$ & -12.6 & $19.8(2.3)$ \\
\hline$(2,0)^{R} Q_{21}(0)$ & $7874:$ & $\leq 5$ & $\ldots$ & $\leq 36$ \\
\hline$(1,0) R_{1}(0)$ & 9186.497 & $52(10)$ & -14.3 & $88(17)$ \\
\hline \multicolumn{5}{|l|}{$\mathrm{BD}+66^{\circ} 1674$} \\
\hline$(2,0) R_{1}(0)$ & 7906.195 & $5.2(0.5)$ & -15.4 & $23.7(2.3)$ \\
\hline$(2,0)^{R} Q_{21}(0)$ & 7874.422 & $3.0(0.5)$ & -16.2 & $21.5(3.6)$ \\
\hline$(1,0) R_{1}(0)$ & 9186.354 & $17(10)$ & -18.9 & $28.7(16.9)$ \\
\hline \multicolumn{5}{|l|}{$\mathrm{BD}+66^{\circ} 1675$} \\
\hline$(2,0) R_{1}(0)$ & $7906.009^{a}$ & $2.4(0.5)$ & -22.4 & $10.8(2.3)$ \\
\hline$(2,0) R_{1}(0)$ & $7906.183^{a}$ & $5.0(0.5)$ & -15.8 & $22.5(2.3)$ \\
\hline$(2,0) R_{1}(0)$ & $7906.365^{a}$ & $3.0(0.5)$ & -8.9 & $13.5(2.3)$ \\
\hline$(2,0) R_{1}(0)$ & $9186.923^{b}$ & $22(10)$ & -0.4 & $37(17)$ \\
\hline$(2,0)^{R} Q_{21}(0)$ & $7874.252^{a}$ & $2.0(0.5)$ & -22.6 & $14.0(3.6)$ \\
\hline$(2,0)^{R} Q_{21}(0)$ & $7874.394^{a}$ & $2.0(0.5)$ & -17.2 & 14.7(3.6) \\
\hline$(2,0)^{R} Q_{21}(0)$ & $7874.591^{a}$ & $2.0(0.5)$ & -9.7 & $14.7(3.6)$ \\
\hline
\end{tabular}

${ }^{a}$ Decomposition not unique.

${ }^{b}$ Blended with telluric line.

the absorption line is treated explicitly, adopting wavelengths of $4300.3030 \AA$ and $4300.3235 \AA$ for the two components. A band oscillator strength of $f_{00}=0.00506$ is used to infer column densities. Columns 2-5 of Table 3 list the heliocentric wavelengths, the equivalent widths, the inferred heliocentric velocities, and the $\mathrm{CH}$ column densities $N_{\infty}$. Measurement uncertainties in $W_{\lambda}$ and corresponding uncertainties in $N_{\infty}$ are given in parentheses. Column 6 of Table 3 gives column densities for $\mathrm{CH}$ inferred in the limit of $b=1 \mathrm{~km} \mathrm{~s}^{-1}$. The uncertainties in $N_{b=1}$ correspond to the measurement uncertainties of $W_{\lambda}$. Towards BD+66 $1669 \mathrm{a}, \mathrm{BD}+66^{\circ} 1674$, and BD $+66^{\circ} 1675$, inferred column densities for $b=1 \mathrm{~km} \mathrm{~s}^{-1}$ are very large indeed. For the latter three stars, the tabulated column densities given in brackets correspond to equivalent widths of $60-65 \mathrm{~m} \AA$. Upper limits in $N(\mathrm{CH})$ which are allowed by the measurement uncertainties are well above $10^{15} \mathrm{~cm}^{-2}$. Table 3 also lists, in Col. 7, $\mathrm{CH}$ column densities $N_{b=2.4}$ inferred for a Doppler parameter of $b=2.4 \mathrm{~km} \mathrm{~s}^{-1}$. The particular value of $b=2.4 \mathrm{~km} \mathrm{~s}^{-1}$ is justified in Sect. 3.1. The large range

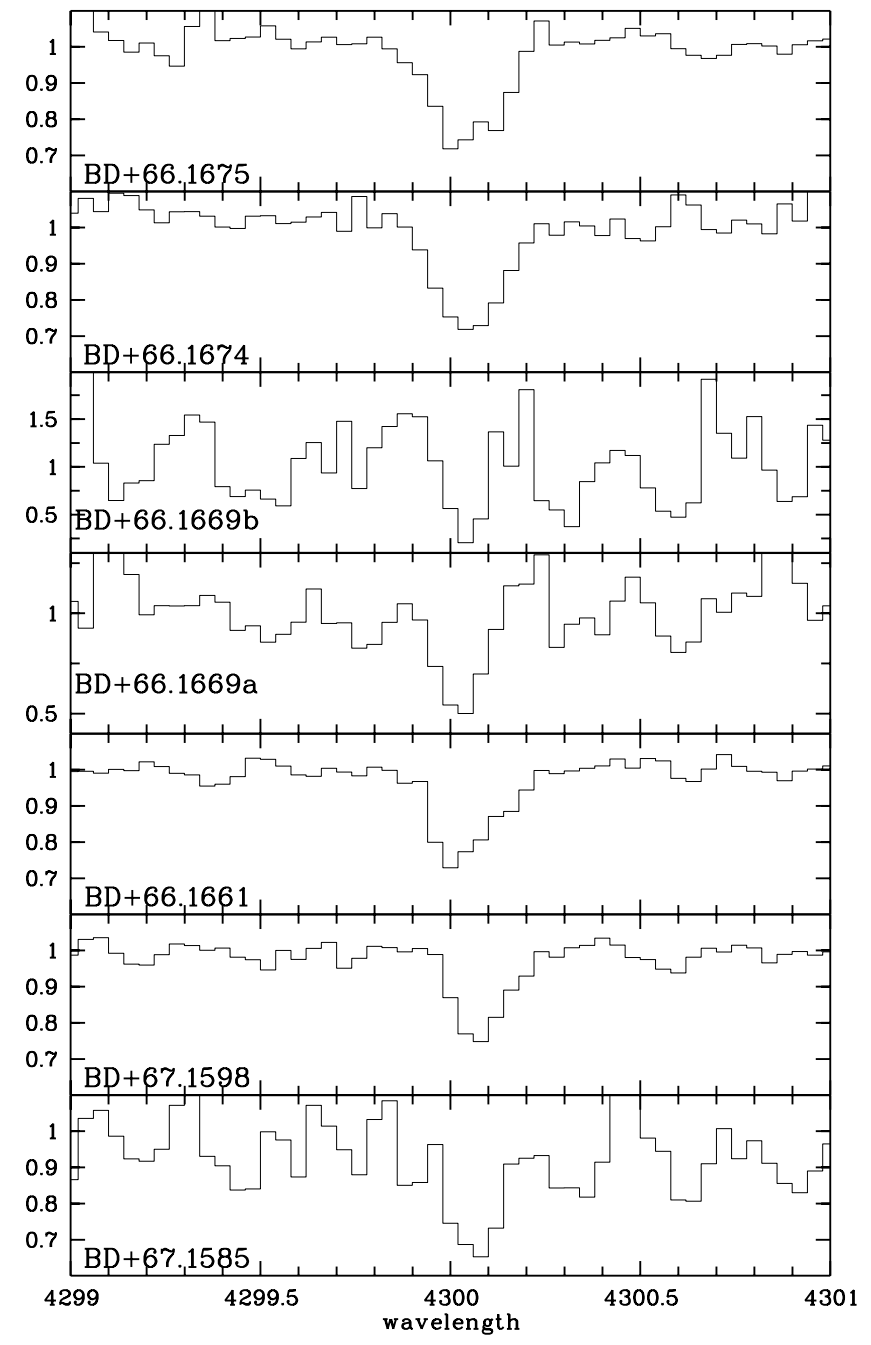

Fig. 2. Normalised spectra of the $R_{2 \mathrm{e}}(1)+R_{2 \mathrm{f}}(1) \Lambda$ doublet of the $(0,0)$ band of the $\mathrm{CH} \mathrm{A}^{2} \Delta-\mathrm{X}^{2} \Pi$ system.

in column densities obtained for $b \rightarrow \infty, b=2.4 \mathrm{~km} \mathrm{~s}^{-1}$, and $b=1 \mathrm{~km} \mathrm{~s}^{-1}$ demonstrate that the largest uncertainties in the $\mathrm{CH}$ column densities arise from the uncertainties in $b$, and not from the measurement uncertainties in $W_{\lambda}$. In the discussion of Sect. 4 , we use $N_{b=2.4}$ as final $\mathrm{CH}$ column densities, and assign uncertainties which reflect the range in column densities from $N_{\infty}$ to $N_{b=1}$.

$\mathrm{CH}^{+}$absorption lines which arise in the $R(0)$ line of the $(0,0)$ band of the $\mathrm{A}^{1} \Pi-\mathrm{X}^{1} \Sigma^{+}$system, near $4232 \AA$, are marginally detected towards four stars. The corresponding spectra are shown in Fig. 3. In the calculation of column densities and heliocentric velocities, rest wavelengths of $4232.548 \AA$ and band oscillator strength of $f_{00}=0.00545$ are used (Gredel et al. 1993). $\mathrm{CH}^{+}$column densities are calculated in the limit of $b \rightarrow \infty$ and for $b=1 \mathrm{~km} \mathrm{~s}^{-1}$. The $\mathrm{CH}^{+}$data are included in Table 3. The detected $\mathrm{CH}^{+}$absorption lines are rather faint and the $\mathrm{CH}^{+}$column densities are not sensitive to Doppler parameters $b>1 \mathrm{~km} \mathrm{~s}^{-1}$. The $R(1)$ and $Q(1)$ lines near $4229 \AA$ and $4237 \AA$, respectively, are not detected. 
Table 3. Measured $\mathrm{CH}$ and $\mathrm{CH}^{+}$lines.

\begin{tabular}{|c|c|c|c|c|c|}
\hline $\begin{array}{r}\lambda_{\text {hel }} \\
\AA\end{array}$ & $\begin{array}{r}W_{\lambda} \\
\mathrm{m} \AA\end{array}$ & $\begin{array}{r}V_{\text {hel }} \\
\mathrm{km} \mathrm{s}^{-1}\end{array}$ & $\begin{array}{r}N_{\infty} \\
10^{13} \mathrm{~cm}^{-2}\end{array}$ & $\begin{array}{r}N_{b=1} \\
10^{13} \mathrm{~cm}^{-2}\end{array}$ & $\begin{array}{r}N_{b=2.4} \\
10^{13} \mathrm{~cm}^{-2}\end{array}$ \\
\hline \multicolumn{6}{|l|}{$\mathrm{BD}+67^{\circ} 1585$} \\
\hline 4300.063 & $44(10)$ & -17.4 & $5.3(1.3)$ & $10.4_{-4}^{+8}$ & $7.1_{-2}^{+3}$ \\
\hline \multicolumn{6}{|c|}{$\mathrm{BD}+67^{\circ} 1598$} \\
\hline 4300.077 & $38(5)$ & -16.4 & $4.6(0.6)$ & $7.8(2.0)$ & $5.8_{-1}^{+2}$ \\
\hline 4232.359 & $15.5(5)$ & -13.3 & $1.8(.6)$ & $2.3(1.0)$ & \\
\hline \multicolumn{6}{|c|}{$\mathrm{BD}+66^{\circ} 1661$} \\
\hline 4300.034 & $49(5)$ & -19.4 & $5.9(0.6)$ & $13_{-3}^{+4.5}$ & $8.2_{-2.3}^{+5}$ \\
\hline 4232.272 & $8.6(5)$ & -19.5 & $1.0(0.6)$ & $1.1(0.7)$ & \\
\hline \multicolumn{6}{|l|}{$\mathrm{BD}+66^{\circ} 1669 \mathrm{a}$} \\
\hline 4300.024 & $62(10)$ & -20.1 & $7.5(1.3)$ & {$[20]$} & $11.8_{-4}^{+8}$ \\
\hline \multicolumn{6}{|c|}{$\mathrm{BD}+66^{\circ} 1669 \mathrm{~b}$} \\
\hline $4300:$ & $\leq 40$ & $\ldots$ & $\leq 4.8$ & & \\
\hline \multicolumn{6}{|l|}{$\mathrm{BD}+66^{\circ} 1673$} \\
\hline $4300:$ & $\leq 6$ & $\ldots$ & $\leq 0.7$ & $\leq 0.8$ & $\leq 0.8$ \\
\hline \multicolumn{6}{|l|}{$\mathrm{BD}+66^{\circ} 1674$} \\
\hline 4300.051 & $60(5)$ & -18.2 & $7.2(0.6)$ & {$[20]$} & $11.2_{-4}^{+9}$ \\
\hline 4232.238 & $12(5)$ & -21.9 & $1.4(0.6)$ & $1.7(0.9)$ & \\
\hline \multicolumn{6}{|l|}{$\mathrm{BD}+66^{\circ} 1675$} \\
\hline 4300.041 & $63(5)$ & -19.0 & $7.6(0.6)$ & {$[20]$} & $12.5_{-5}^{+8}$ \\
\hline 4232.27 & $10(5)$ & -19.5 & $1.2(0.6)$ & $1.3(0.9)$ & \\
\hline \multicolumn{6}{|l|}{$\mathrm{BD}+65^{\circ} 1973$} \\
\hline $4300:$ & $\leq 20$ & $\ldots$ & $\leq 2.4$ & $\leq 3.0$ & $\leq 3.0$ \\
\hline
\end{tabular}

\subsection{The (1, 0), $(2,0)$, and $(3,0)$ bands of the $C_{2}$ Phillips system}

The spectra towards $\mathrm{BD}+66^{\circ} 1661, \mathrm{BD}+66^{\circ} 1674$, and $\mathrm{BD}+66^{\circ} 1675$ show prominent $\mathrm{C}_{2}$ absorption lines which arise in the $(2,0)$ band of the $\mathrm{A}^{1} \Pi_{\mathrm{u}}-\mathrm{X}^{1} \Sigma_{\mathrm{g}}^{+}$Phillips system, at wavelengths around $8765 \AA$. The corresponding spectra are shown in Fig. 4. Towards BD+66 1661 , the detected $C_{2}$ lines are explicity identified. Towards all three stars, additional $\mathrm{C}_{2}$ absorption lines which arise in the $(1,0)$ and $(3,0)$ bands, around wavelengths of $10150 \AA$ and $7720 \AA$, respectively, are detected as well. A few $C_{2}$ absorption lines in the $(2,0)$ band are present towards the star $\mathrm{BD}+66^{\circ} 1673$. A summary of the $\mathrm{C}_{2}$ measurements is given in Table 4, with heliocentric wavelengths, measured equivalent widths, heliocentric velocities, and column densities $N\left(J^{\prime \prime}\right)$ listed in Cols. 2-5, respectively. Column 6 of Table 4 lists the average column densities $\left\langle N\left(J^{\prime \prime}\right)\right\rangle$ in $J^{\prime \prime}$ in cases where multiple measurements are available. Rest wavelengths and band oscillator strengths are adopted from Bakker et al. (1997).

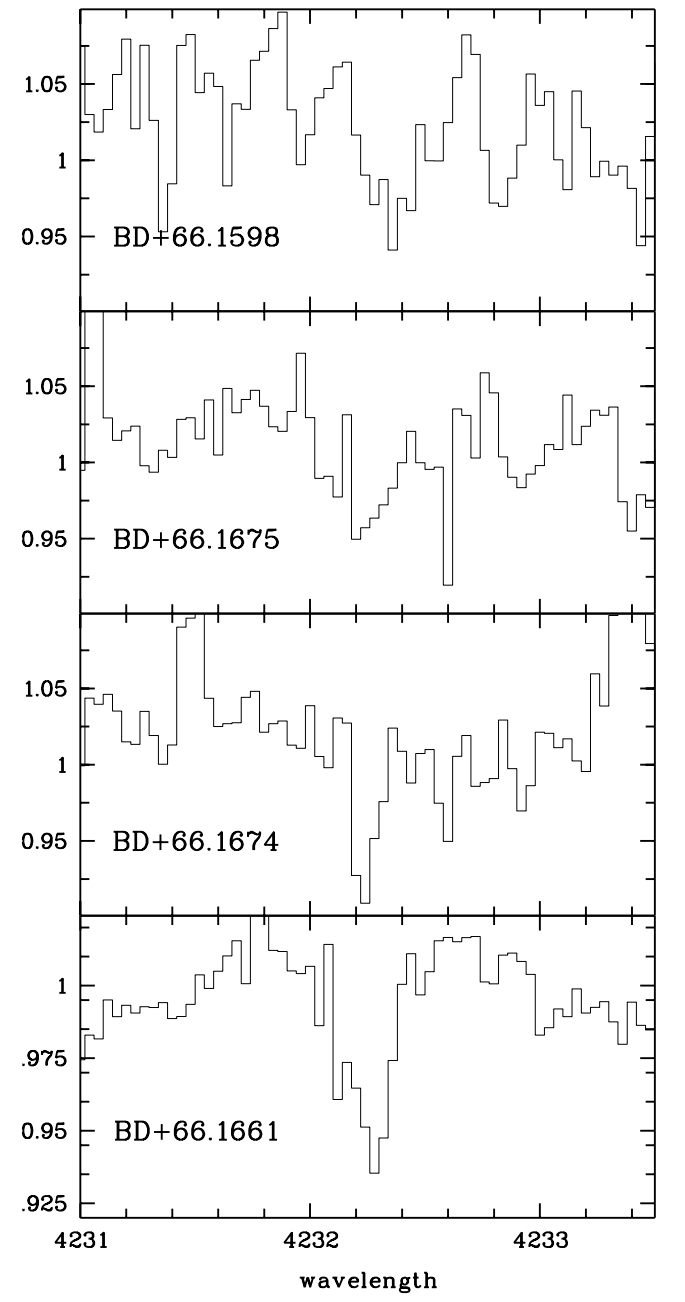

Fig. 3. Normalised spectra of the $\mathrm{CH}^{+} R(0)$ line of the $(0,0)$ band of the $\mathrm{A}^{1} \Pi-\mathrm{X}^{1} \Sigma^{+}$system.

\section{Analysis}

\subsection{The $C N$ Doppler parameter $b(C N)$}

The reported equivalent width of $W_{\lambda}=80 \mathrm{~m} \AA$ for the $\left(R_{1}(0)+\right.$ $\left.{ }^{R} Q_{21}(0)\right)$ absorption line blend of the $(0,0)$ band of the CN violet system by Münch (1964) can be used to infer the $\mathrm{CN}$ Doppler parameter $b(\mathrm{CN})$. If saturation is negligible, the column density in $J^{\prime \prime}=0$ inferred from the measurement of Münch (1964) is $N(0)=1.77 \times 10^{13} \mathrm{~cm}^{-2}$. Our measurements yield an average value of $N(0)=4.5 \times 10^{13} \mathrm{~cm}^{-2}$. We thus perform a curve of growth analysis in order to determine an effective Doppler $b$ parameter for $\mathrm{CN}$ which brings the two results for $N(0)$ into agreement. If the $\mathrm{CN}$ absorption arises in a single velocity component, a value of $b(\mathrm{CN})=2.4 \mathrm{~km} \mathrm{~s}^{-1}$ increases the column density inferred from the $\mathrm{CN}$ violet system to our value of $N(0)=4.5 \times 10^{13} \mathrm{~cm}^{-2}$. If we assume that the $\mathrm{CN}$ absorption arises in three velocity components with velocity separations of some $5 \mathrm{~km} \mathrm{~s}^{-1}$ and $7 \mathrm{~km} \mathrm{~s}^{-1}$ from the central line at its average velocity of $V_{\text {hel }}=-16.5 \mathrm{~km} \mathrm{~s}^{-1}$ (cf. Table 2), a CN Doppler parameter of $b=1.2-1.3 \mathrm{~km} \mathrm{~s}^{-1}$ for each velocity component is obtained. We note that the photographic measurements of Münch (1964) seem to underestimate 


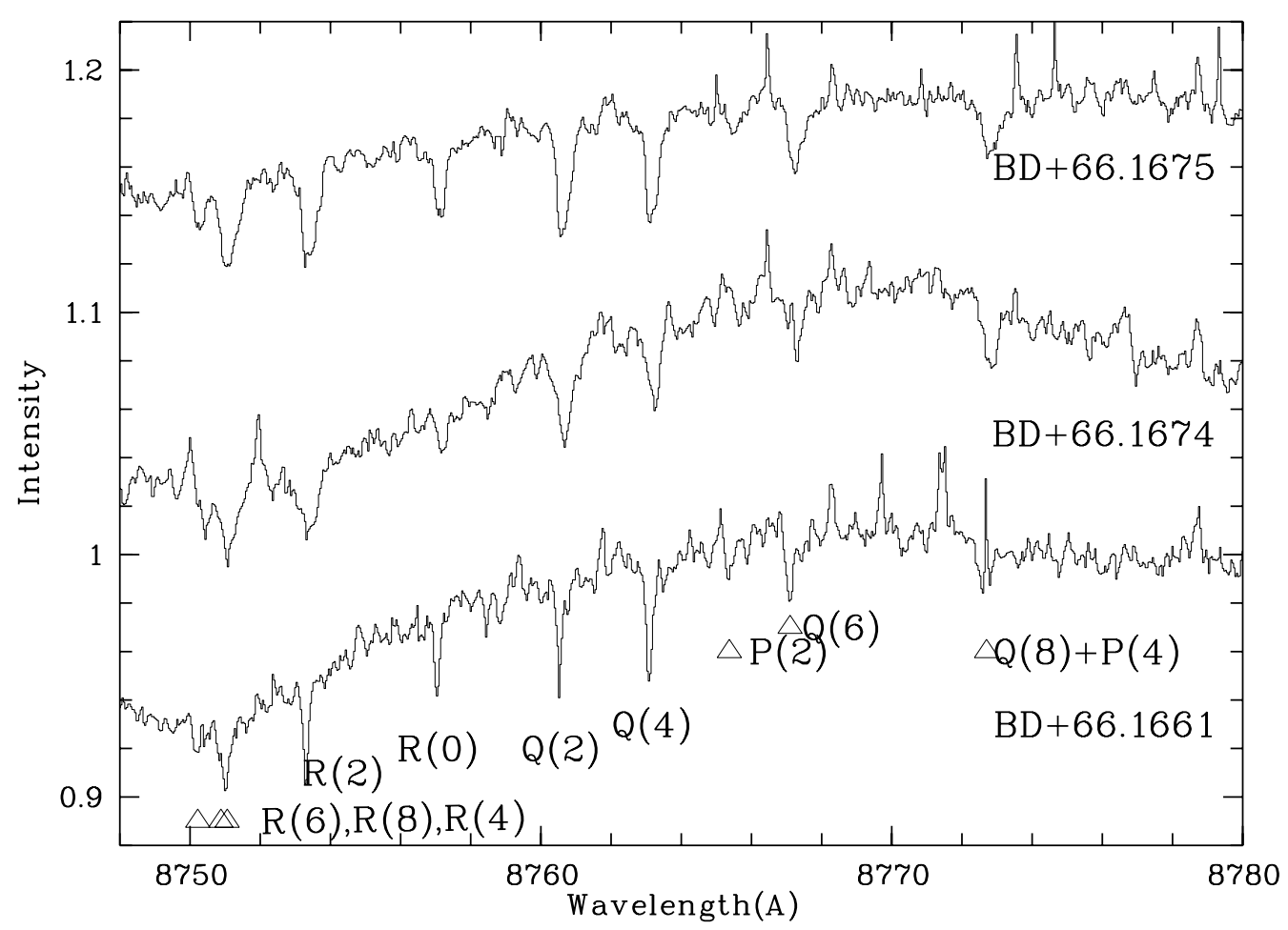

Fig. 4. Absorption lines which arise from the $(2,0)$ band of the $C_{2} A^{1} \Pi_{u}-X^{1} \Sigma_{g}^{+}$Phillips system. The ordinate is valid for $B D+66^{\circ} 1661$ only. Spectra of $\mathrm{BD}+66^{\circ} 1674$, and $\mathrm{BD}+66^{\circ} 1675$, are shifted by units of 0.1 , and 0.2 , respectively, along the ordinate. Detected $\mathrm{C}_{2}$ absorption lines are identified.

the equivalent widths of the absorption lines (see Sect. 4.2). If so, the CN Doppler parameters inferred from the present analysis would increase.

\subsection{Rotational excitation of $\mathrm{CN}$}

As discussed in detail by Black \& van Dishoeck (1991), the rotational excitation of $\mathrm{CN}$ is dominated by radiative interactions with the cosmic background radiation, neutral collisions, and electron impact excitations. Thus, the determination of accurate column densities in the rotational levels $J^{\prime \prime}>1$ allows to gain insight into physical parameters such as total hydrogen and electron densities. Münch (1964) did report the presence of $\mathrm{CN}$ absorption lines from rotational levels up to $J^{\prime \prime}=3$ towards $\mathrm{BD}+66^{\circ} 1675$. In the spectra shown obtained here, $\mathrm{CN}$ absorption lines which arise from rotational levels $J^{\prime \prime} \geq 1$ are absent. The expected position of the $R_{2}(1)+{ }^{R} Q_{21}(1)$ blend, the $R_{1}$ (1) line, the ${ }^{Q} R_{12}(1)+Q_{1}(1)$ blend in the $(2,0)$ band, at heliocentric wavelengths around 7873.4 $\AA$, 7903.3 $\AA$ and 7908.3 $\AA$, respectively, and the $(1,0) R_{1}(1)$ line near $9183 \AA$, are indicated in Fig. 1 by open triangles. Column densities in $N(1)$ inferred from upper limits of $W_{\lambda} \approx 1 \mathrm{~m} \AA$ of the corresponding lines can be used to infer upper limits of the $\mathrm{CN}$ rotational excitation temperature $T_{10}$. We use $T_{10}=5.4419 / \ln (3 N(0) / N(1)$ and obtain $T_{10}<3 \mathrm{~K}$. We proceed to calculate total $\mathrm{CN}$ column densities $N_{\text {tot }}=\Sigma_{J^{\prime \prime}} N\left(J^{\prime \prime}\right)$ using average values of $N(0)$ from the measurements and theoretical column densities $N(1)$ and $N(2)$ derived from an excitation temperature of $T_{10}=2.8 \mathrm{~K}$. Total $\mathrm{CN}$ column densities obtained this way are given in Table 5.

\subsection{Rotational excitation of $C_{2}$}

The analysis of interstellar absorption lines of $\mathrm{C}_{2}$ allow to infer physical parameters in the clouds where the molecules form. The method developed by van Dishoeck \& Black (1982) is routinely adopted to infer gaskinetic temperatures and densities in homogeneous translucent molecular clouds (e.g. Gredel et al. 2001, and references therein). The method benefits from the fact that in translucent clouds, in general, the population density in the lowest rotational levels is dominated by collisions, and thus thermalised, while the population density in the higher rotational levels may deviate from a thermal distribution because of radiative pumping. The deviations from a thermal population distribution among the higher rotational levels allow to determine the ratio $n_{\mathrm{c}} / I$, where $n_{\mathrm{c}}$ is the density of the collision partners $\left(n_{\mathrm{c}}=n(\mathrm{H})+n\left(\mathrm{H}_{2}\right)\right)$ and $I$ is the scaling factor of the interstellar radiation field.

Figure 5 contains the $\mathrm{C}_{2}$ excitation diagrams constructed for the three stars $\mathrm{BD}+66^{\circ} 1661, \mathrm{BD}+66^{\circ} 1674$, and $\mathrm{BD}+66^{\circ} 1675$, with values of $-\ln \left\{5 N\left(J^{\prime \prime}\right) /\left(2 J^{\prime \prime}+\right.\right.$ 1) $\left./ 10^{13} \mathrm{~cm}^{-2}\right\}$ plotted versus excitation energies $E\left(J^{\prime \prime}\right)$ of rotational levels $J^{\prime \prime}$. In the three diagrams, data points inferred from $(2,0)$ band observations are represented by open triangles, while open squares and open pentagons refer to data obtained from $(3,0)$ and $(1,0)$ band measurements, respectively. In cases where various determinations of $N\left(J^{\prime \prime}\right)$ are available for a given $J^{\prime \prime}$, arithmetic means are plotted and are represented by filled squares.

In the excitation diagram of $\mathrm{BD}+66^{\circ} 1661$, the theoretical $\mathrm{C}_{2}$ population distributions obtained for $T_{\text {kin }}=35 \mathrm{~K}$ and 
Table 4. Measured $\mathrm{C}_{2}$ lines.

\begin{tabular}{|c|c|c|c|c|}
\hline $\begin{array}{l}\text { Star and } \\
\text { line ID }\end{array}$ & $\begin{array}{r}\lambda_{\text {hel }} \\
\AA\end{array}$ & $\begin{array}{c}W_{\lambda} \quad V_{\mathrm{he}} \\
\mathrm{m} \AA \mathrm{km} \mathrm{s}^{-1}\end{array}$ & $\begin{array}{r}N\left(J^{\prime \prime}\right) \\
10^{13} \mathrm{~cm}^{-2}\end{array}$ & $\begin{array}{r}\left\langle N\left(J^{\prime \prime}\right)\right\rangle \\
10^{13} \mathrm{~cm}^{-2}\end{array}$ \\
\hline
\end{tabular}

$\mathrm{BD}+66^{\circ} 1661$

$(2,0) R(0)$

$(2,0) R(2)$

$(3,0) R(2)$

$(2,0) Q(2)$

$(3,0) Q(2)$

$(2,0) P(2)$

$(2,0) R(4)$

$(3,0) R(4)$

$(2,0) Q(4)$

$(3,0) Q(4)$

$(2,0) P(4)$

$(2,0) R(6)$

$(2,0) Q(6)$

$(2,0) R(8)$

$(2,0) Q(8)$

$\mathrm{BD}+66^{\circ} 1673$
$(2,0) R(0)$
$(2,0) R(2)$
$(2,0) R(4)$
$(2,0) Q(4)$

$\mathrm{BD}+66^{\circ} 1674$

$(2,0) R(0)$

$(2,0) R(2)$

$(3,0) R(2)$

$(1,0) Q(2)$

$(2,0) Q(2)$

$(2,0) R(4)$

$(3,0) R(4)$

$(2,0) Q(4)$

$(2,0) P(4)$

$(2,0) R(6)$

$(2,0) Q(6)$

$(2,0) Q(8)$

$(2,0) R(10)$

$\mathrm{BD}+66^{\circ} 1675$
$(2,0) R(0)$
$(2,0) R(2)$
$(3,0) R(2)$
$(1,0) Q(2)$
$(2,0) Q(2)$
$(2,0) P(2)$
$(2,0) R(4)$
$(2,0) Q(4)$
$(2,0) P(4)$
$(2,0) R(6)$
$(1,0) Q(6)$
$(2,0) Q(6)$
$(2,0) R(8)$
$(2,0) Q(8)$

$\begin{array}{llll}8757.042 & 5.9(1.0) & -22.0 & 0.5(0.1) \\ 8753.299 & 8.4(1.0) & -22.2 & 1.8(0.2) \\ 7715.958 & 3.2(0.5) & -22.1 & 2.0(0.3) \\ 8760.531 & 6.6(1.0) & -22.6 & 1.1(0.2) \\ 7721.505 & 8.5(0.5) & -22.9 & 4.2(0.2) \\ 8765.369 & 2.4(1.0) & -22.6 & 2.1(0.9) \\ 8751.060 & 6.2(1.0) & -21.4 & 1.6(0.3) \\ 7714.347 & 1.5(0.5) & -23.2 & 1.1(0.4) \\ 8763.086 & 8.7(1.0) & -22.7 & 1.5(0.2) \\ 7723.655 & 3.6(0.5) & -21.8 & 1.8(0.2) \\ 8772.746 & 3.6(1.0) & -23.3 & 1.9(0.5) \\ 8750.221 & 3.1(1.0) & -21.5 & 0.9(0.3) \\ 8767.100 & 6.2(1.0) & -22.5 & 1.1(0.2) \\ 8750.880 & 3.6(1.0) & -20.7 & 1.1(0.3) \\ 8772.647 & 3.6(1.0) & -19.6 & 0.6(0.2)\end{array}$

$0.5(0.1)$

2.25
1.6

1.0
0.85

0.85

$\begin{array}{llll}8757.311 & 4.8(2.0) & -12.8 & 0.4(0.2)\end{array}$ $\begin{array}{llll}8753.556 & 4.5(2.0) & -13.4 & 1.0(0.4)\end{array}$ $8751.266 \quad 10.5(2.0) \quad-14.3 \quad 2.7(0.6)$ $8763.372 \quad 11.7(2.0) \quad-12.9 \quad 2.0(0.4)$
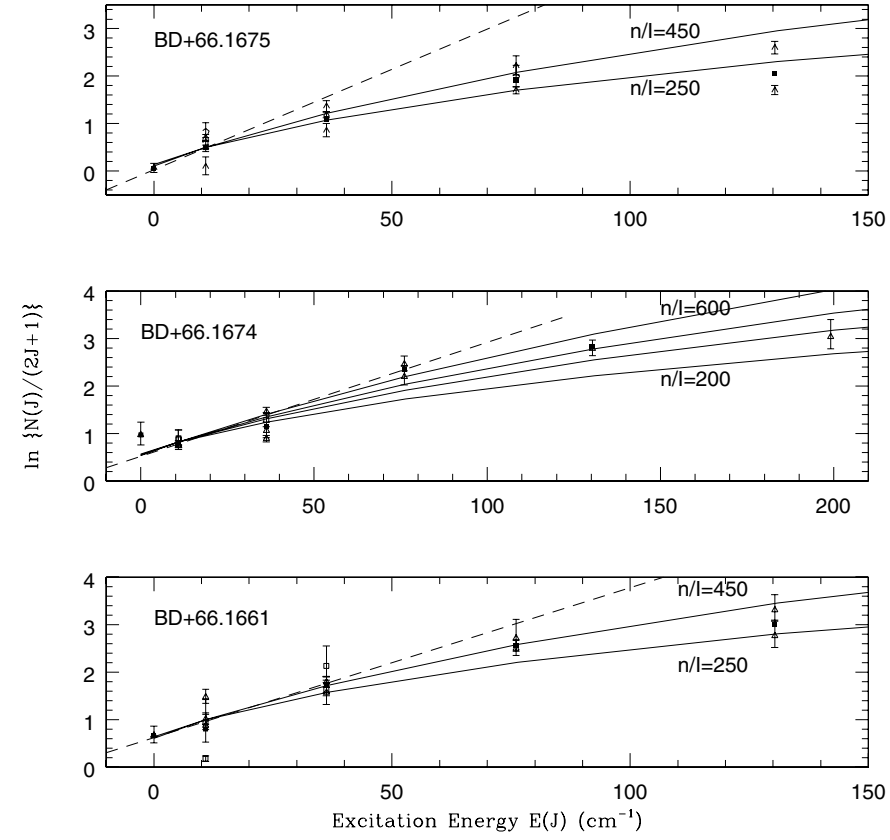

Fig. 5. $\mathrm{C}_{2}$ excitation diagrams towards $\mathrm{BD}+66^{\circ} 1661, \mathrm{BD}+66^{\circ} 1674$, and $\mathrm{BD}+66^{\circ} 1675$. Plotted are values of $\ln \left(N\left(J^{\prime \prime}\right) /\left(2 J^{\prime \prime}+1\right) / 10^{13} \mathrm{~cm}^{-2}\right)$ versus rotational excitation energies $E\left(J^{\prime \prime}\right)$. Filled lines correspond to theoretical population distributions (see text). Dashed lines correspond to thermal population distributions obtained for gaskinetic temperatures of $T_{\text {kin }}=35 \mathrm{~K}$ for $\mathrm{BD}+66^{\circ} 1661$ and $\mathrm{BD}+66^{\circ} 1675$ and for $T_{\text {kin }}=60 \mathrm{~K}$ for $\mathrm{BD}+66^{\circ} 1674$.
0.4

$\begin{array}{lll}4.4(1.0) & -16.6 & 0.4(0.1) \\ 17.4 & 2.4(0.2)\end{array}$

$\begin{array}{llll}7716.112 & 3.3(0.5) & -16.1 & 2.0(0.3)\end{array}$

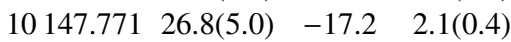

$\begin{array}{llll}8760.688 & 13.4(1.0) & -17.3 & 2.3(0.2)\end{array}$

$8751.097 \quad 14.2(1.0) \quad-20.1 \quad 3.7(0.3)$

$\begin{array}{llll}7714.560 & 3.4(0.5) & -14.9 & 2.5(0.4)\end{array}$

$8763.225 \quad 12.0(1.0) \quad-18.0 \quad 2.1(0.2)$

$\begin{array}{llll}8772.822 & 6.0(1.0) & -20.7 & 3.1(0.5)\end{array}$

$\begin{array}{llll}8750.451 & 5.1(1.0) & -13.6 & 1.4(0.3)\end{array}$

$8767.317 \quad 6.4(1.0) \quad-15.1 \quad 1.1(0.2)$

$\begin{array}{llll}8772.822 & 6.0(1.0) & -13.6 & 1.0(0.2)\end{array}$

$8753.118 \quad 3.3(1.0) \quad-15.7 \quad 1.0(0.3)$ parameters $n_{\mathrm{c}} / I=250 \mathrm{~cm}^{-3}$ and $450 \mathrm{~cm}^{-3}$ are represented by full lines. The dashed line corresponds to a thermal population distribution at $T_{\text {kin }}=35 \mathrm{~K}$. The parameter range of $n_{\mathrm{c}} / I=$ $350 \pm 100 \mathrm{~cm}^{-3}$ does provide a good fit to the observations. From the theoretical models, total $\mathrm{C}_{2}$ population densities $N_{\text {tot }}=\Sigma_{J^{\prime \prime}} N\left(J^{\prime \prime}\right)$ are obtained, where the summation is carried out over rotational levels in the vibrational state $v^{\prime \prime}=0$. For $T_{\text {kin }}=35 \mathrm{~K}$ and $n_{\mathrm{c}} / I=250 \mathrm{~cm}^{-3}, N_{\text {tot }}=4.9 \times N(2)$, and for $n_{\mathrm{c}} / I=450 \mathrm{~cm}^{-3}, N_{\mathrm{tot}}=3.4 \times N(2)$.

The full lines in the excitation diagram of $\mathrm{BD}+66^{\circ} 1674$ correspond to nonthermal population distributions characterised by $T_{\text {kin }}=60 \mathrm{~K}$ and values of $n_{\mathrm{c}} / I=200,300,400$, and $600 \mathrm{~cm}^{-3}$. The dashed lines corresponds to a thermal distribution at $T_{\text {kin }}=60 \mathrm{~K}$. The measurements are consistent with densities in the range of $n_{\mathrm{c}} / I=400 \pm 200 \mathrm{~cm}^{-3}$. Corresponding total $\mathrm{C}_{2}$ column densities are $N_{\text {tot }}=7.1 \times N(2)$ for $n_{\mathrm{c}} / I=$ $200 \mathrm{~cm}^{-3}$ and $N_{\mathrm{tot}}=4.0 \times N(2)$ for $n_{\mathrm{c}} / I=600 \mathrm{~cm}^{-3}$. A more rigorous constraint of the densities requires the measurement of population densities in rotational levels $J^{\prime \prime}>10$ which is not available. Note that the inferred population density in $J^{\prime \prime}=0$ towards $\mathrm{BD}+66^{\circ} 1674$ is too low if compared to $N(2)$ or $N(4)$. The cause for the weakness of the $R(0)$ line remains unclear after a re-analysis of the data, but it does not have a physical origin.

The full lines in the excitation diagram towards $\mathrm{BD}+66^{\circ} 1675$ characterise nonthermal population distributions obtained for $n_{\mathrm{c}} / I=250 \mathrm{~cm}^{-3}$ and $450 \mathrm{~cm}^{-3}$ and $T_{\mathrm{kin}}=$ $35 \mathrm{~K}$. The corresponding thermal distribution at $T_{\text {kin }}=35 \mathrm{~K}$ 
is indicated by the dashed line. The observed population distribution is well delimited by this parameter range.

Towards the three stars, the $\mathrm{C}_{2}$ data indicate relatively low gaskinetic temperatures in the $35-60 \mathrm{~K}$ range, and parameters of $n_{\mathrm{c}} / I \leq 600 \mathrm{~cm}^{-3}$. Uncertainties in the derived gaskinetic temperatures are of the order of $10 \mathrm{~K}$. It is argued in Sect. 4 that the molecular gas seen in absorption towards Cep OB4 is probably not located in the vicinity of the stars. We thus adopt a scaling factor of the interstellar radiation field not larger than $I=1$. In the presence of pure molecular gas, the $\mathrm{C}_{2}$ analysis indicates total hydrogen densities $n=n(\mathrm{H})+2 n\left(\mathrm{H}_{2}\right)$ of $n=$ $700 \pm 200 \mathrm{~cm}^{-3}$ toward BD $+66^{\circ} 1661, n=800 \pm 400 \mathrm{~cm}^{-3}$ toward $\mathrm{BD}+66^{\circ} 1674$, and $n=700 \pm 200 \mathrm{~cm}^{-3}$ toward $\mathrm{BD}+66^{\circ} 1675$. The corresponding total $\mathrm{C}_{2}$ population densities are $N_{\text {tot }}=(9.3 \pm 1.7) \times 10^{13} \mathrm{~cm}^{-2}$ toward BD+66 1661 , $N_{\text {tot }}=(11.5 \pm 2.8) \times 10^{13} \mathrm{~cm}^{-2}$ toward BD $+66^{\circ} 1674$, and $N_{\text {tot }}=$ $(12.7 \pm 2.2) \times 10^{13} \mathrm{~cm}^{-2}$ toward $\mathrm{BD}+66^{\circ} 1675$. The uncertainties correspond to the range in $n_{\mathrm{c}} / I$ which is derived from the excitation diagrams of Fig. 5.

The few $\mathrm{C}_{2}$ measurements towards $\mathrm{BD}+66^{\circ} 1673$ do not allow to construct a useful $\mathrm{C}_{2}$ excitation diagram. The scatter in the inferred rotational population distributions is too large to infer a robust temperature or density estimate.

In their analysis of $\mathrm{C}_{2}$ observations towards $\mathrm{Cyg}$ OB2 No. 12, Cecchi-Pestellini \& Dalgarno (2002) showed that the average excitation temperatures which are obtained from the assumption of a homogeneous cloud can also be obtained from a two-component cloud model where dense clumps are embedded into a lower-density gas with filling factors of 0.1-0.2. In particular, they showed that $\mathrm{C}_{2}$ is not sensitive to smallscale density fluctuations. In their model, Cecchi-Pestellini \& Dalgarno (2002) showed that the observed $\mathrm{C}_{2}$ population distribution towards Cyg OB2 No. 12 is well fitted by a twophase model where dense cores of $n \approx 10^{4} \mathrm{~cm}^{-3}$ are embedded into a low-density medium. The standard analysis in terms of a single, homogeneous cloud results in $T_{\mathrm{ex}}=35 \mathrm{~K}$ and $n=600 \pm 100 \mathrm{~cm}^{-3}$ (Gredel et al. 2001). The concept of single translucent clouds towards heavily reddened lines of sight has been questioned altogether by Rachford et al. (2002). In their analysis of FUSE data towards stars with visual reddening up to $A_{\mathrm{V}}=2.5 \mathrm{mag}$, the authors found little evidence for the presence of individual translucent clouds towards the sightlines studied, and concluded that their data is better described in terms of the presence of several diffuse clouds along the line of sight. The physical parameters inferred from the present $\mathrm{C}_{2}$ analysis should thus be used with care.

\section{Discussion}

Observations of the association designated as Cepheus IV by Alter et al. (1958) have first been reported by Blanco \& Williams (1959) and MacConnell (1968). We follow the recommendations of the IAU and use the nomenclature of Ruprecht et al. (1982) to name OB associations. The stars studied here belong to Cep OB4, and are located at Galactic coordinates of $l=118, b=5.20$. Note that until June 2004, a search in SIMBAD related Cepheus IV with Cep OB5. Cep OB5, in the standard nomenclature, is located at Galactic coordinates of $l=108, b=-2.75$. The correct identification is now available in SIMBAD as well.

The results of the analysis described in the previous section are summarised in Table 5. For $\mathrm{C}_{2}$, Cols. 2-5 list the inferred gaskinetic temperatures $T_{\text {kin }}$, gas densities $n$, total observed column densities $N_{\text {obs }}$, and total theoretical column densities $N_{\text {tot }}$ obtained from the best fit non-thermal models. Columns 6 and 7 list average $\mathrm{CN}$ observed column densities $N(0)$ and total column densities assuming an excitation temperature of $2.8 \mathrm{~K}$. Columns 8 and 9 list $\mathrm{CH}$ and $\mathrm{CH}^{+}$column densities. Average radial velocities of $\mathrm{C}_{2}, \mathrm{CN}, \mathrm{CH}$, and $\mathrm{CH}^{+}$are given in Cols. 10-13, respectively. The $\mathrm{CH}$ measurements towards $\mathrm{BD}+67^{\circ} 1585$ and $\mathrm{BD}+66^{\circ} 1669 \mathrm{a}, \mathrm{b}$ are given in Table 3 and are not repeated in Table 5. In general, the radial velocities of $\mathrm{CH}, \mathrm{CH}^{+}, \mathrm{C}_{2}$, and $\mathrm{CN}$ agree very well with each other. Velocity uncertainties for individual velocity determinations are of the order of $1-1.5 \mathrm{~km} \mathrm{~s}^{-1}$.

\subsection{The velocity structure of molecular gas towards Cep OB4}

A mean radial LSR velocity of $V_{\mathrm{LSR}}=-14.5 \mathrm{~km} \mathrm{~s}^{-1}$ has been obtained by Liu et al. (1989) for Berkeley 59, the central star cluster of Cep OB4. The authors assign the rather large velocity uncertainty of $8 \mathrm{~km} \mathrm{~s}^{-1}$ because two out of the three stars of their sample, $\mathrm{BD}+66^{\circ} 1674$ and $\mathrm{BD}+66^{\circ} 1675$, are spectroscopic binaries, and the third star, $\mathrm{BD}+66^{\circ} 9487$, has but one measurement. From their observations of the largescale structure of the neutral gas, Rossano et al. (1983) found three individual velocity components of $\mathrm{H}_{2} \mathrm{CO}$ at $\mathrm{LSR}$ velocities of $-13.2,-7.1$, and $-1 \mathrm{~km} \mathrm{~s}^{-1}$. The $-13 \mathrm{~km} \mathrm{~s}^{-1}$ cloud spreads in velocity from -11.8 to $-15.1 \mathrm{~km} \mathrm{~s}^{-1}$. Emission in $\mathrm{H} 110 \alpha$ appears near velocities of $V_{\mathrm{LSR}}=-14.3,-9.0$, and $+1.2 \mathrm{~km} \mathrm{~s}^{-1}$. Millimeter emission of ${ }^{13} \mathrm{CO}$ was observed by Liu et al. (1988), who found a strong emission component at $V_{\mathrm{LSR}}=-14.4 \mathrm{~km} \mathrm{~s}^{-1}$ and two weaker components near $V_{\mathrm{LSR}}=-7.0 \mathrm{~km} \mathrm{~s}^{-1}$ and $-0.4 \mathrm{~km} \mathrm{~s}^{-1}$. The two "clouds" which correspond to the velocity components at $-14.4 \mathrm{~km} \mathrm{~s}^{-1}$ and $-7.0 \mathrm{~km} \mathrm{~s}^{-1}$, respectively, are diametrically opposed to Berkeley 59, which led Liu et al. (1988) to speculate that they are the fossil remnants of a bi-polar outflow (cf. their Fig. 6). The physical connection of the two velocity components was however questioned by Yang \& Fukui (1992), who assigned the component near $-7 \mathrm{~km} \mathrm{~s}^{-1}$ to foreground molecular gas. $\mathrm{CO}$ emission around $-7 \mathrm{~km} \mathrm{~s}^{-1}$ is seen over some 100 square degrees and shows a good coincidence with weak extinction features on the POSS plates. The gas seen at $-14.4 \mathrm{~km} \mathrm{~s}^{-1}$ arises from two dense molecular clumps denoted $\mathrm{C} 1$ and $\mathrm{C} 2$, and Yang \& Fukui (1992) concluded that both clumps are in physical contact with the HII region formed by Berkeley 59.

Towards $\mathrm{BD}+66^{\circ} 1674$ and $\mathrm{BD}+66^{\circ} 1675$, which are both members of Berkeley 59, the molecular absorption lines observed here appear at a mean heliocentric velocity of $V_{\text {hel }}=-18.35 \mathrm{~km} \mathrm{~s}^{-1}$, which corresponds to $V_{\mathrm{LSR}}=-9 \mathrm{~km} \mathrm{~s}^{-1}$. The LSR velocities of the foreground gas seen in emission ranges from $-7 \mathrm{~km} \mathrm{~s}^{-1}$ to $-10 \mathrm{~km} \mathrm{~s}^{-1}$ (cf. Fig. 3 of Yang \& Fukui 1992). The agreement in radial velocities suggests that 
Table 5. Summary of observations.

\begin{tabular}{|c|c|c|c|c|c|c|c|c|c|c|c|c|}
\hline \multirow[t]{2}{*}{ Star } & \multicolumn{4}{|c|}{$\mathrm{C}_{2}$} & \multicolumn{2}{|r|}{$\mathrm{CN}$} & \multirow{2}{*}{$\begin{array}{r}\mathrm{CH} \\
N\end{array}$} & \multirow{2}{*}{$\begin{array}{r}\mathrm{CH}^{+} \\
\quad N\end{array}$} & \multirow{2}{*}{$\begin{array}{c}\mathrm{C}_{2} \\
V_{\text {hel }}\end{array}$} & \multirow{2}{*}{$\begin{array}{l}\mathrm{CN} \\
V_{\text {hel }}\end{array}$} & \multirow{2}{*}{$\begin{array}{l}\mathrm{CH} \\
V_{\text {hel }}\end{array}$} & \multirow{2}{*}{$\begin{array}{r}\mathrm{CH}^{+} \\
V_{\text {hel }}\end{array}$} \\
\hline & $T_{\text {kin }}$ & $n$ & $N_{\text {obs }}$ & $N_{\text {tot }}$ & $N(0)$ & $N_{\text {tot }}$ & & & & & & \\
\hline $\mathrm{BD}+67^{\circ} 1598$ & $\ldots$ & & $\ldots$ & $\ldots$ & 7.9 & $1.15(0.2)$ & $5.8_{-1}^{+2}$ & $2.3(1.0)$ & & -14.1 & -16.4 & -13.3 \\
\hline $\mathrm{BD}+66^{\circ} 1661$ & $35 \pm 10$ & $700 \pm 200$ & 6.2 & $9.3 \pm 1.7$ & 30.3 & $4.40(0.5)$ & $8.2_{-2.3}^{+5}$ & $1.1(0.7)$ & -22.1 & -23.3 & -19.4 & -19.5 \\
\hline $\mathrm{BD}+66^{\circ} 1673$ & $\ldots$ & $\ldots$ & 3.75 & $\ldots$ & 19.8 & $2.87(0.5)$ & $\leq 3$ & $\ldots$ & -13.4 & -12.6 & $\ldots$ & $\ldots$ \\
\hline $\mathrm{BD}+66^{\circ} 1674$ & $60 \pm 10$ & $800 \pm 400$ & 8.7 & $11.5 \pm 2.8$ & 24.6 & $3.57(0.5)$ & $11.2_{-4}^{+9}$ & $1.7(0.9)$ & -16.6 & -16.8 & -18.2 & -21.9 \\
\hline $\mathrm{BD}+66^{\circ} 1675$ & $35 \pm 10$ & $700 \pm 200$ & 11.05 & $12.7 \pm 2.2$ & 45.1 & $6.54(0.5)$ & $12.5_{-5}^{+8}$ & $1.3(0.9)$ & -18.7 & -16.1 & -19.0 & -19.5 \\
\hline
\end{tabular}

$T_{\text {kin }}$ in $\mathrm{K} ; n$ in $\mathrm{cm}^{-3} ; N_{\text {obs }}, N_{\text {tot }}, N$ in $10^{13} \mathrm{~cm}^{-2} ; V_{\text {hel }}$ in $\mathrm{km} \mathrm{s}^{-1}$.

the gas seen in absorption is spatially coexistent with this gas component. Based on its kinematics, it is thus unlikely that the bulk of the $\mathrm{CN}, \mathrm{C}_{2}, \mathrm{CH}$, and $\mathrm{CH}^{+}$absorption arises in the PDR which is located at LSR velocities of $-14 \mathrm{~km} \mathrm{~s}^{-1}$. The possibility exists, however, than a minor fraction of the $\mathrm{CN}$ absorption arises in the $\mathrm{HII}-\mathrm{H}_{2}$ interaction region near Berkeley 59. In Sect. 2.1 a very uncertain decomposition of the $\mathrm{CN}$ absorption line towards $\mathrm{BD}+66^{\circ} 1675$ into three velocity components has been described. The corresponding LSR velocities are $V_{\mathrm{LSR}}=-13.2,-7$, and $0 \mathrm{~km} \mathrm{~s}^{-1}$. The component near $-13.2 \mathrm{~km} \mathrm{~s}^{-1}$ holds about $25 \%$ of the total equivalent width. It can thus not be ruled out that a minor fraction of the gas seen in absorption towards $\mathrm{BD}+66^{\circ} 1675$ arises in molecular material close to the star, although the evidence is rather poor.

\subsection{Earlier measurements}

The measurements reported here agree in general with the earlier data towards Cep OB4. The heliocentric velocity $V_{\text {hel }}=-18.8 \mathrm{~km} \mathrm{~s}^{-1}$ for $\mathrm{CN}$ towards $\mathrm{BD}+66^{\circ} 1675$ inferred by Münch (1964) is consistent with our value of $V_{\text {hel }}=-16.1 \mathrm{~km} \mathrm{~s}^{-1}$. Towards BD $+66^{\circ} 1674$, both Münch (1964) and the present study report a mean $\mathrm{CN}$ heliocentric velocity of $V_{\text {hel }}=-16.8 \mathrm{~km} \mathrm{~s}^{-1}$. Somewhat discrepant are the $\mathrm{CN}$ velocities towards $\mathrm{BD}+66^{\circ} 1661$, where our value of $-23.3 \mathrm{~km} \mathrm{~s}^{-1}$ contrasts the value of $-17.7 \mathrm{~km} \mathrm{~s}^{-1}$ of Münch (1964). The detection of diatomic carbon towards $\mathrm{BD}+66^{\circ} 1675$ has been reported by Lutz \& Crutcher (1983). While the assignation of $\mathrm{C}_{2}$ absorption features in their spectrum requires some courage, their measurements indicate a $C_{2}$ column density of $(21 \pm 12) \times 10^{13} \mathrm{~cm}^{-2}$ when scaled to the $f_{1,0}$ oscillator strength used here, while we find $(12.7 \pm 2.2) \times 10^{13} \mathrm{~cm}^{-3}$. The reported equivalent width of $12.3 \pm 6.2 \mathrm{~m} \AA$ for the $(1,0) Q(2)$ line is close to our value of $14.4 \pm 1 \mathrm{~m} \AA$. The inferred temperature of $71 \pm$ $37 \mathrm{~K}$ for the $\mathrm{C}_{2}$ rotational excitation of Lutz \& Crutcher (1983) is consistent with our value of $T_{\text {kin }}=35 \pm 10 \mathrm{~K}$. Towards BD $+66^{\circ} 1675$, the medium-resolution MMT observations of van Dishoeck \& Black (1989) indicate the presence of a double-component $\mathrm{CH}$ absorption profile with a total $\mathrm{CH}$ column density of $N(\mathrm{CH})=(15 \pm 5) \times 10^{13} \mathrm{~cm}^{-2}$. Their measured equivalent with of $W_{\lambda}=(72 \pm 8) \mathrm{m} \AA$ is in good agreement with our value of $63 \pm 10 \mathrm{~m} \AA$. In his work, Münch (1964) cites a value of about $W_{\lambda}=40 \mathrm{~m} \AA$ for the $\mathrm{CH}$ absorption line towards $\mathrm{BD}+66^{\circ} 1675$, which is significantly lower than the equivalent widths inferred with electronic detectors. The discrepancy may indicate that the use of photographic plates led to an underestimation of the equivalent widths of the absorption lines towards Cep OB4.

\subsection{Chemical abundances towards Cep OB4}

Figures $6 \mathrm{a}-\mathrm{d}$ contain plots of the observed column densities of $\mathrm{CH}^{+}, \mathrm{CH}, \mathrm{C}_{2}$, and $\mathrm{CN}$ versus the reddening $E_{B-V}$ of the background star. None of the column densities are correlated with the reddening. In quiescent, translucent clouds, the $\mathrm{CH}$ column density is in general tightly correlated with $E_{B-V}$ (cf. Liszt \& Lucas 2002, and references therein), and there are indications that in general, the column densities of $\mathrm{CN}, \mathrm{C}_{2}$, and $\mathrm{CH}^{+}$increase with with $E_{B-V}$ as well (e.g. Federman et al. 1994; Gredel 1997). The absence of correlations of molecular column densities with the reddening of the background stars may indicate that the lines of sight towards Cep OB4 trace large amounts of dust associated with low density, atomic material. It may also indicate that the molecular abundances peak in a thin layer of the cloud, in which case the total molecular column densities are unrelated with the total optical depth of the cloud. For diatomic molecules such as $\mathrm{CH}, \mathrm{CH}^{+}$and $\mathrm{CN}$, this behaviour is expected in photon dominated regions. Photon-dominated regions or PDRs form when molecular material is illuminated by a strong radiation field from nearby stars. The chemistry and physical characteristics of dense PDRs have been reviewed by Hollenbach \& Tielens (1997). The chemistry of dense PDRs differs from that of dense molecular clouds in various ways. At the surface of PDRs, large abundances of molecules which have main formation routes via photoreactions are expected because of the strong ultraviolet radiation field which is present up to optical depths of a few. The UV photons maintain a large fraction of atomic hydrogen in the $\mathrm{HII}-\mathrm{H}_{2}$ transition layer, thus reactions which involve atomic hydrogen are important as well.

The millimeter emission line observations of Yang \& Fukui (1992) have resulted in the detection of two dense molecular clumps with a total mass of $600 M_{\odot}$ near Berkeley 59, and the authors suggested that the star cluster generates an 

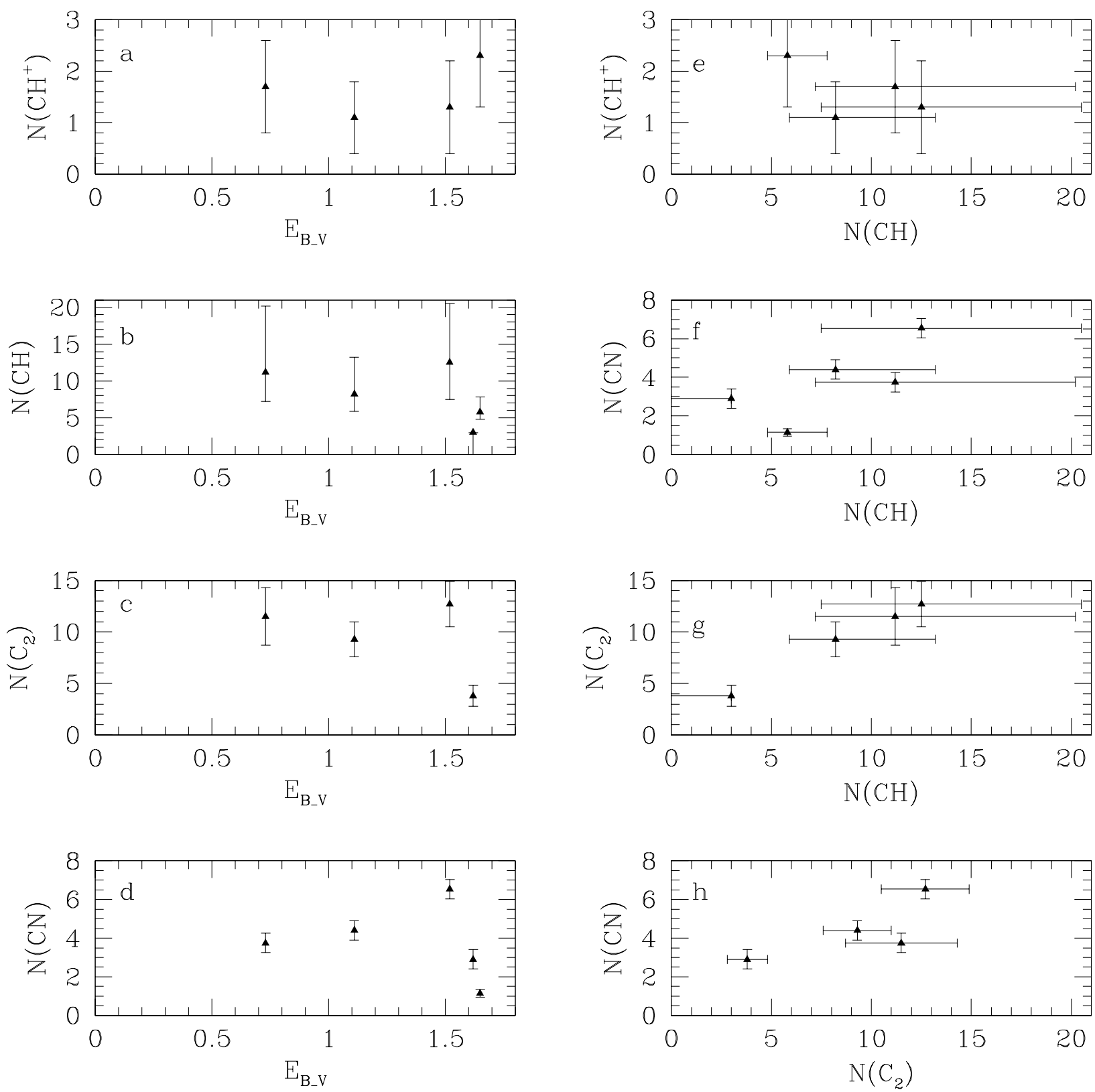

Fig. 6. a)-d): Molecular column densities of $\mathrm{CH}^{+} \mathrm{CH}, \mathrm{C}_{2}$, and $\mathrm{CN}$, in units of $10^{13} \mathrm{~cm}^{-2}$, plotted versus reddening $E_{B-V}$ (in magnitudes). e)-f) Molecular column densities of $\mathrm{CH}^{+}, \mathrm{CN}$, and $\mathrm{C}_{2}$ plotted versus molecular column density of $\mathrm{CH}$, in units of $10^{13} \mathrm{~cm}^{-2}$. h) $\mathrm{Column}$ density of $\mathrm{CN}$ plotted versus column density of $\mathrm{C}_{2}$, both in units of $10^{13} \mathrm{~cm}^{-2}$.

ionisation front on the surface of the clumps. If the dense clumps are located in the foreground of the star cluster, the interstellar molecular absorption lines studied here may very well arise in in the outer layers of a PDR. In their comprehensive chemical model of a dense PDR, Sternberg \& Dalgarno (1995) showed that the $\mathrm{CN}$ abundances peak at the relatively low optical depths of about $A_{\mathrm{V}}=2 \mathrm{mag}$. Their model is characterised by a density of $n=10^{6} \mathrm{~cm}^{-3}$ and an intensity of the incident FUV radiation field a factors of $\chi=2 \times 10^{6}$ stronger than the ambient interstellar radiation field. The largest abundance of $\mathrm{CN}$ occurs in the $\mathrm{H} / \mathrm{H}_{2}$ transition layer and falls off rapidly at higher depths into the PDR, where the temperature decreases rapidly (cf. Fig. 7 of Sternberg \& Dalgarno 1995). For background stars with sightlines passing through the outer layers of a dense PDR, the CN column densities would thus be constant towards stars with $A_{\mathrm{V}}>2$ mag. The abundance variation of $\mathrm{CH}$ and $\mathrm{CH}^{+}$shows a similar behaviour. The PDRs produce very large $\mathrm{CH}$ and $\mathrm{CH}^{+}$abundances in a thin outer layer, which peak at the relatively low optical depths of $A_{\mathrm{V}} \leq 0.5$ mag. At larger depths, fractional abundances fall off rapidly, and integrated column densities are constant for visual extinctions of $A_{\mathrm{V}}>2 \mathrm{mag}$, or $E_{B-V} \leq 0.7 \mathrm{mag}$.

The qualitative agreement between the present observations and the theoretical predictions of the PDR model is not matched, however, by the quantitative predictions of the model. Maximal integrated CN column densities of the model of Sternberg \& Dalgarno (1995) are $N(\mathrm{CN}) \leq 10^{13} \mathrm{~cm}^{-2}$, which is about an order of magnitude lower than what is 
observed here. In addition, PDRs produce very large amounts of $\mathrm{CH}$ and $\mathrm{CH}^{+}$, compared to quiescent translucent clouds. Total integrated column densities are $N(\mathrm{CH}) \approx 10^{15} \mathrm{~cm}^{-2}$ and $N\left(\mathrm{CH}^{+}\right) \approx 10^{14} \mathrm{~cm}^{-2}$, or about an order of magnitude larger than observed $\mathrm{CH}$ and $\mathrm{CH}^{+}$column densities. It is thus unlikely that the molecules observed here arise in the outer layer of a dense PDR. This result is in agreement with the analysis of the velocity structure of Sect. 4.1, which on kinematic grounds indicates that the foreground molecular material is not in physical contact with the HII region produced by Berkeley 59 .

Mid- to far-infrared emission line observations of atomic fine structure lines using ISO have been reported by Okada et al. (2003). The authors collected one dimensional raster scans starting at the position of Berkeley 59 out to some $5 \mathrm{pc}$ away from the cluster. From the emission line observations, which also trace material in the background of the stars of Berkeley 59, the molecular part of the PDR formed by the star cluster was detected. As it is the case for other PDRs as well, it was found that the observed [OI] $63 \mu \mathrm{m} /[\mathrm{OI}] 146 \mu \mathrm{m}$ ratio was too small if compared with the predictions of the more recent PDR models of Kaufman et al. (1999). In order to explain the small line ratio, the authors suggested that self-absorption of the [OI] emission line by foreground gas along the line of sight occurs. Self-absorption of the [OI] $63 \mu \mathrm{m}$ line has been used to explain the low [OI] $63 \mu \mathrm{m} /[\mathrm{OI}] 146 \mu \mathrm{m}$ line ratio in NGC 2024, and is seen in high spectral resolution KAO observations towards star forming regions (see Okada et al. 2003 for references). The proposition by Okada et al. (2003) provides further support to the suggestion that cold molecular gas exists towards Berkeley 59 which is detached from the PDR created by the star cluster.

The processes which lead to the formation of interstellar $\mathrm{CN}$ in quiescent translucent clouds have been identified some 20 years ago (see Federman et al. 1994, and references therein). In translucent molecular clouds, $\mathrm{CN}$ probes the denser gas in general, and $\mathrm{CN}$ column densities are only poorly correlated with the reddening of the background stars. On the other side, $N(\mathrm{CN})$ is in general well correlated with $N\left(\mathrm{C}_{2}\right)$, as shown by van Dishoeck \& Black (1989) and Federman et al. (1994). The tight $N(\mathrm{CN})-N\left(\mathrm{C}_{2}\right)$ correlation which has been inferred towards other regions is also present in our data, as seen in Fig. 6h. Since the formation and destruction channels of both molecules involve different dependencies on the optical depth $\tau_{\mathrm{UV}}$ in the ultraviolet, the correlation of the column densities is not expected on chemical grounds but reflects the fact that both molecules are formed in similar parcels of gas, as suggested by Federman et al. (1994). Our data are consistent with the relation $\log N(\mathrm{CN})=1.56 \log N\left(\mathrm{C}_{2}\right)$ of Federman et al. (1994).

In Fig. 6f, the column densities of $\mathrm{CN}$ are plotted versus the column density of $\mathrm{CH}$. Despite the large uncertainties which hamper the derivation of accurate column densities, a few correlations are obvious from the figures. The data are consistent with the relation $N(\mathrm{CN}) \approx 0.5 N(\mathrm{CH})$. The formation of interstellar $\mathrm{C}_{2}$ in translucent clouds has been discussed in Federman \& Huntress (1989). The derived column densities $N(\mathrm{CN})$ are tightly correlated with $N\left(\mathrm{C}_{2}\right)$. The data are consistent with $N\left(\mathrm{C}_{2}\right)=N(\mathrm{CH})$ (cf. Fig. $6 \mathrm{~g}$ ). The values given in Table 5 follow the empirical relations of van Dishoeck \& Black (1989) and the theoretical relation of Federman et al. (1994). In translucent molecular clouds, the CH column density appears to be a good measure of the molecular hydrogen column density, and Liszt \& Lucas (2002) find a mean relation of $N(\mathrm{CH}) / N\left(\mathrm{H}_{2}\right)=(4.3 \pm 1.9) \times 10^{-8}$. The $\mathrm{CH}$ column densities may thus be used to derive fractional abundances $f(X)=N(X) / 2 N(\mathrm{H})=N(X) / 2 N\left(\mathrm{H}_{2}\right)$ of species $X$ assuming that all hydrogen is in molecular form. This procedure fixes the fractional abundance of $\mathrm{CH}$ to $f(\mathrm{CH})=2.15 \times 10^{-8}$ for all lines of sight. Our measurements indicate fractional abundances of $f(\mathrm{CN})=0.4-2.9 \times 10^{-8}, f\left(\mathrm{C}_{2}\right)=2.2-3.6 \times 10^{-8}$, and $f\left(\mathrm{CH}^{+}\right)=2.2-8.5 \times 10^{-9}$. The derived fractional abundances of $\mathrm{CN}$ and $\mathrm{CH}$ are very typical for translucent molecular clouds and are well fit by the models of van Dishoeck \& Black (1989).

The marginal data on $\mathrm{CH}^{+}$obtained here are not useful to provide new contributions to the discussion related to the formation scenarios of interstellar $\mathrm{CH}^{+}$. The column densities of $\mathrm{CH}^{+}$are not correlated with $N(\mathrm{CH})$, as can be seen from Fig. 6e. The derived $\mathrm{CH}^{+}$column densities are relatively small if compared to values of $N\left(\mathrm{CH}^{+}\right)$inferred towards other stars with similar reddenings. This may indicate that the dominant $\mathrm{CH}^{+}$formation processes which are at work towards other OB associations are not efficient towards Cep OB4.

\section{Summary}

We have analysed interstellar absorption lines of $\mathrm{CN}, \mathrm{C}_{2}, \mathrm{CH}$, and $\mathrm{CH}^{+}$towards stars in the Cep OB4 association and its central cluster Berkeley 59. The present observations provide no evidence to support an earlier suggestion that the large column densities of interstellar $\mathrm{CN}$ towards $\mathrm{BD}+66^{\circ} 1661$, $\mathrm{BD}+66^{\circ} 1674$, and $\mathrm{BD}+66^{\circ} 1675$, originate in the $\mathrm{HII}-\mathrm{H}_{2}$ interface region near Berkeley 59. The analysis of the velocity structure of the molecular gas, the molecular abundances of $\mathrm{CH}, \mathrm{CH}^{+}$, and indirect evidence from far-infrared atomic emission line observations, suggest that the $\mathrm{CN}, \mathrm{C}_{2}, \mathrm{CH}$, and $\mathrm{CH}^{+}$absorption lines arise in molecular material well detached from the PDR formed by the central cluster Berkeley 59. Our treatment of the $\mathrm{C}_{2}$ excitation results in gaskinetic temperatures of $35-60 \mathrm{~K}$ and densities of $700-800 \mathrm{~cm}^{-3}$ in the foreground translucent cloud. Total molecular column densities of $\mathrm{CN}$ and $\mathrm{C}_{2}$ are tightly correlated with the column density of $\mathrm{CH}$. The correlations, and the column densities of $\mathrm{CN}, \mathrm{C}_{2}$, and $\mathrm{CH}$, are well understood in terms of models of quiescent translucent clouds.

Acknowledgements. Part of the observations have been carried out in service mode by Calar Alto staff. It is a pleasure to thank the Calar Alto astronomy group for their support.

\section{References}

Andersson, B.-G., Wannier, P. G., \& Crawford, I. A. 2002, MNRAS, 334,327

Alter, G., Ruprecht, J., \& Vanýsek, V. 1958, Catalogue of Star Clusters and Associations (Praque: Czechoslovak Academy of Sciences)

Bakker, E. J., van Dishoeck, E. F., Waters, L. B. F. M., \& Schoenmaker, T. 1997, A\&A, 323, 469 
Black, J. H., \& van Dishoeck, E. F. 1991, ApJ, 369, L9

Blanco, V. M., \& Williams, A. D. 1959, ApJ, 130, 482

Cecchi-Pestellini, C., \& Dalgarno, A. 2002, MNRAS, 331, L31

Crawford, I. A. 1995, MNRAS, 277, 458

de Zeeuw, P. T., Hoogerwerf, R., de Bruijne, J. H. J., Brown, A. G. A., \& Blaauw, A. 1999, AJ, 117, 354

Federman, S. R., \& Huntress, W. T., Jr. 1989, ApJ, 338, 140

Federman, S. R., Strom, C. J., Lambert, D. L., et al. 1994, ApJ, 424, 772

Gredel, R. 1997, A\&A, 320, 929

Gredel, R., Black, J. H., \& Yan, M. 2001, A\&A, 375, 553

Gredel, R., van Dishoeck, E. F., \& Black, J. H. 1991, A\&A, 251, 625

Gredel, R., van Dishoeck, E. F., \& Black, J. H. 1993, A\&A, 269, 477

Hollenbach, D. J., \& Tielens, A. G. G. M. 1997, ARA\&A, 35, 179

Grupp, F. 2003, A\&A, 412, 897

Kaufman, M. J., Wolfire, M. G., Hollenbach, D. J., \& Luhman, M. L. 1999, ApJ, 527, 795

Liszt, H., \& Lucas, R. 2002, A\&A, 391, 693

Liu, T., Janes, K. A., Bania, T. M., \& Phelps, R. L. 1988, AJ, 95, 1122
Liu, T., Janes, K. A., \& Bania, T. M. 1989, AJ, 98, 626

Luo, S.-G. 1995, ChA\&A, 19, 316

Lutz, B. L., \& Crutcher, R. M. 1983, ApJ, 271, L101

MacConnell, D. J. 1968, ApJS, 16, 275

Münch, G. 1964, ApJ, 140, 107

Okada, Y., Onaka, T., Shibai, H., \& Doi, Y. 2003, A\&A, 412, 199

Pfeiffer, M. J., Frank, C., Baumüller, D., Fuhrmann, K., \& Gehren, T. 1998, A\&AS, 130, 381

Rachford, B. L, Snow, T. P., Tumlinson, J., et al. 2002, ApJ, 577, 221

Rossano, G. S., Grayzeck, E. J., \& Angerhofer, P. E. 1983, AJ, 88, 1835

Ruprecht, J., Balazs, B., \& White, R. E. 1982, Star Clusters and Associations, VizieR On-line Data Catalog: VII/31B (originally published in 1982, BICDS, 22, 132)

Sternberg, A., \& Dalgarno, A. 1995, ApJS, 99, 565

van Dishoeck, E. F., \& Black, J. H. 1982, ApJ, 258, 533

van Dishoeck, E. F., \& Black, J. H. 1989, ApJ, 340, 273

Walker, G. A. H. 1965, ApJ, 141, 660

Yang, J., \& Fukui, Y. 1992, ApJ, 386, 618 